\section{International Scientific Journal Theoretical \& Applied Science}

\author{
p-ISSN: 2308-4944 (print) e-ISSN: 2409-0085 (online) \\ Year: $2014 \quad$ Issue: $11 \quad$ Volume: 19 \\ Published: $30.11 .2014 \quad$ http://www.T-Science.org
}

\author{
Andrey Alexandrovich Kokolov \\ Junior research associate, Ph.D. \\ Tomsk State University of Control Systems and \\ Radioelectronics, Russia \\ kokolovaa@gmail.com
}

SECTION 26. Radio-technique. Electronics.

Telecommunications.

\title{
MATHEMATICAL MODELS FOR MICROWAVE FET
}

Abstract: Currently there are a huge number of field effect transistors nonlinear models, many of which apply to the High-electron-mobility transistor HEMT. In this article a review and classification of existing nonlinear models of microwave HEMT is performed. This review will be useful to engineers involved in the design of microwave devices to make the choice of microwave transistor models.

Key words: nonlinear model, microwave FET, HEMT.

Language: Russian

Citation: Kokolov AA (2014) MATHEMATICAL MODELS FOR MICROWAVE FET. ISJ Theoretical \& Applied Science 11 (19): 70-76. doi: http://dx.doi.org/10.15863/TAS.2014.11.19.14

\section{МАТЕМАТИЧЕСКИЕ МОДЕЛИ СВЧ ПОЛЕВЫХ ТРАНЗИСТОРОВ}

Аннотация: В настоящее время существует огромное количество моделей полевых транзисторов, многие из которых применимы к транзисторам с высокой подвижность электронов (НЕМТ). В данной работе произведен обзор и классификация существующих моделей СВЧ НЕМТ-транзисторов. Данный обзор будет полезен инженерам, занимающимся проектированием СВЧ устройств, при выборе типа модели СВЧ транзистора.

Ключевые слова: нелинейная модель, СВЧ полевой транзистор, НЕМТ.

Введение. Разработка СВЧ схем значительно ускоряется и удешевляется при использовании систем автоматизированного проектирования (САПР). Однако, для этого нужны соответствующие математические модели активных элементов (АЭ), описывающие нелинейности, высокочастотный шум, саморазогрев и другие факторы. Целью данной работы является обзор и классификация существующих моделей СВЧ полевых транзисторов с высокой подвижностью электронов (HEMT - High Electron Mobility Transistor), в частности для pHEMT (псевдморфоные НЕMT) и (метаморфные НЕMT).

В настоящее время существует огромное число моделей полевых транзисторов (ПТ), многие из которых применимы к транзисторам с высокой подвижностью электронов. Модели, получившие наибольшее распространение среди разработчиков СВЧ устройств интегрированы в современные САПР. Например, в AWR MWO 2013 встроены 39 нелинейных моделей ПТ. Некоторые САПР допускают возможность использования моделей, написанных самим пользователем, либо на стандартном языке описания моделей Verilog, либо с использованием специального дополнительного программного продукта (SDK - Software Design Kit), который интегрируют написанную на $\mathrm{C} / \mathrm{C}++$ модель в программу моделирования. При таком разнообразии неудивительно, что перед разработчиком стоит проблема выбора наиболее подходящей для проектирования модели, которая бы с достаточной точность отражала параметры рассеяния, нелинейные эффекты, шумы и т.п.

Исследователи делят существующие математические модели на несколько групп (рис. $1)$, в зависимости от степени абстракции $[1,2]$ :

1) Физико-технологические модели (отражают физические процессы, происходящие в полупроводнике, основаны на решении системы дифференциальных уравнений в частных производных).

2) Компактные модели (модели в виде эквивалентной схемы, которая описывает поведение транзистора относительно его внешних зажимов). 
3) Поведенческие модели (используются различные математические функции, системы дифференциальных уравнений или специальные преобразования для связи выходных данных с входными).
4) Аппаратные модели (строятся в виде специализированного устройства, в котором информация о модели отражена в структуре электрических связей между его электрическими блоками).

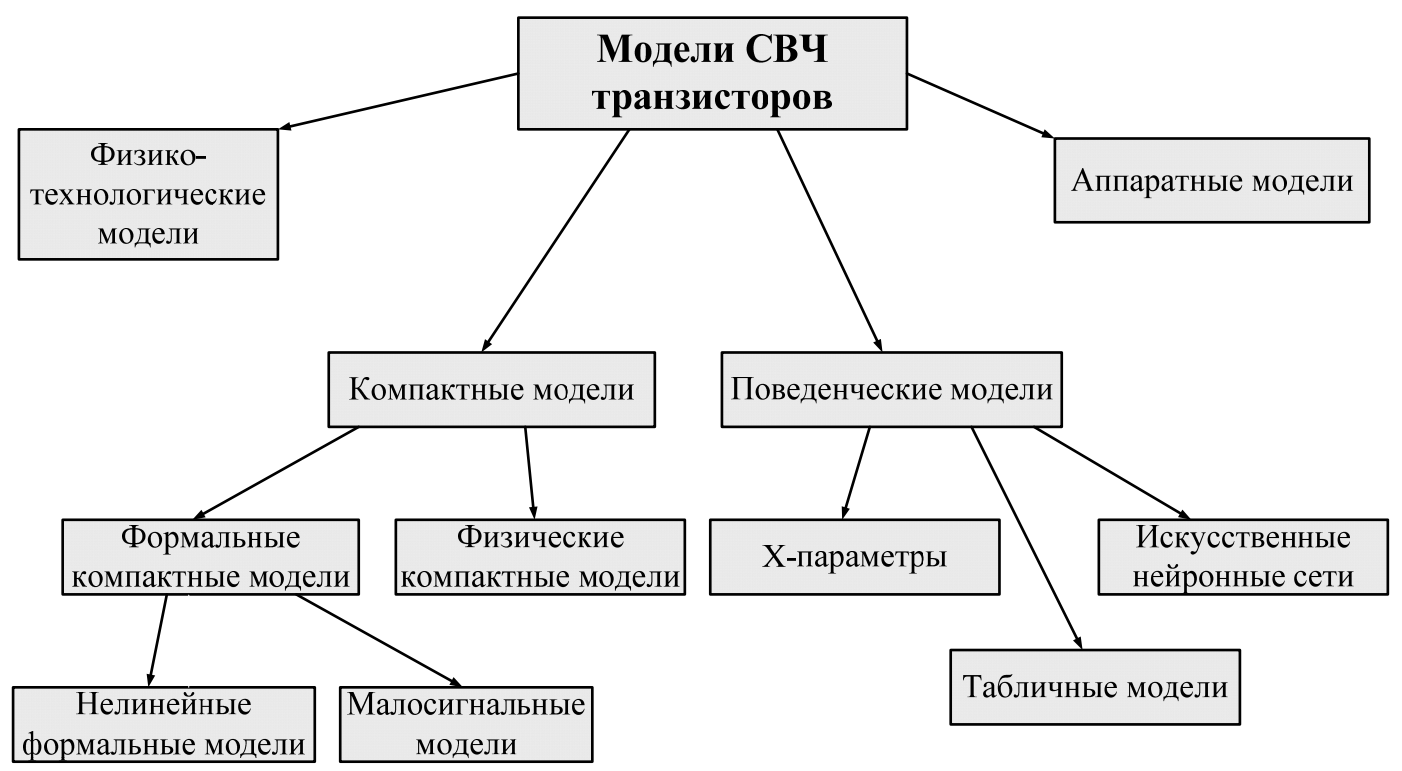

Рисунок 1 - Классификация математических моделей транзисторов.

В физико-технологических моделях при моделировании структуры транзистора решаются нелинейные дифференциальные уравнения в частных производных, которые описывают распределение заряда, перенос заряда, непрерывность тока, квантово-механические эффекты и т.д. При моделировании физическая структура транзистора разбивается на мелкие части в двумерном или трехмерном пространстве и производится решение дифференциальных уравнений в частных производных и квантовомеханических уравнений для каждой элементарной ячейки или узла структуры при помощи метода конечных разностей [3] или метода конечных элементов [4]. Данные численные методы используются в коммерческом программном обеспечении для физикотехнологического моделирования (технологические CAПP - TCAD), например: ISE и Silvaco.

Недостатками физико-технологического подхода являются значительное время моделирования и необходимость знания очень большого количества электрофизических параметров моделируемого прибора для его правильного расчета. Это означает, что физикотехнологическое моделирование обычно непригодно для решения задач схемотехнического проектирования. Однако данная методика успешно применяется в технологическом цикле разработки для проектирования новых транзисторов. При физико-технологическом моделировании можно воспроизвести вольтамперные характеристики и параметры рассеяния, зависимые от смещения, зная только физические свойства материалов и геометрические размеры СВЧ транзистора. Эти данные затем можно использовать для того, чтобы узнать, как надо изменить материалов и конструкции исследуемого полупроводникового прибора, чтобы улучшить его ВЧ характеристики.

Компактные модели, или модели в виде эквивалентных схем (ЭС), наиболее часто используются при разработке СВЧ схем и на сегодняшний момент составляют большую часть из моделей, представленных в САПР. Термин «компактная модель» является общепризнанным в зарубежной литературе и отражает основное требование к таким моделям - вычислительная простота (компактность) [2].

Главное отличительное свойство компактных моделей - это возможность применения в САПР для моделирования радиоэлектронных устройств на уровне электрических схем. Современные схемы содержат множество элементов, при этом часто приходится производить оптимизацию параметров элементов. Поэтому при разработке компактной модели приходится преодолевать противоречие между ее точностью и 
быстродействием. В случае проектирования активных СВЧ устройств компактные модели полупроводниковых приборов, как правило, представляют собой модели в виде электрических эквивалентных схем. Определенное распространение получил также термин «структурные модели», он подчеркивает, что модель некоторым образом отражает внутреннюю структуру и физические особенности исходного объекта, в отличие от так называемых «бесструктурных», или поведенческих моделей [5]. В частности, ЭС СВЧ транзистора на электрическом уровне описывает определенные свойства активной структуры (сопротивления и емкости переходов, усилительные свойства т.д.), а также паразитные емкости и индуктивности соединительных выводов.

В отечественной литературе компактные модели называют также электрическими моделями или компонентными моделями [5]. Компактные модели делятся на физические и формальные; некоторые исследователи выделяют в отдельный класс табличные модели.

Формальные компактные модели строятся на основе подбора экспертным путем аналитических функций, описывающих нелинейные свойства транзистора только в зависимости от электрических переменных например, напряжений на зажимах транзистора (Angelov [6], Materka [7], Curtice [8], EEHEMT [9] и т.п.). Критерием подбора зачастую является наилучшее соответствие измеренных и смоделированных характеристик транзистора. При этом не учитывается влияние на характеристики элемента, например, параметров того или иного технологического процесса, материалов или топологии. В связи с этим указанные модели можно назвать также «компактными электрическими моделями».

Недостатком таких моделей является то, что способ подбора нелинейных функций и их параметров не формализован и обычно основан либо на первичном расчете параметров и дальнейшей оптимизации [10], либо на использовании специализированного программного обеспечении [11]. К достоинствам относятся высокая скорость моделирования при достаточной для проектирования точности, наличие программного обеспечения для расчета параметров модели, а также широкое распространение компактных моделей среди инженеров.

Компактные формальные модели делят на линейные (малосигнальные) и нелинейные. Линейная модель (рис. 2) верна только для одного режима по постоянному току в активном режиме, при этом предполагается, что изменения переменного тока и напряжения в окрестности рабочей точки сравнительно небольшие [12]. Элементы линейной модели не зависят от амплитуды входного переменного напряжения. В нелинейных моделях параметры элементов ЭС являются некоторыми функциями напряжений, температуры и т.д.

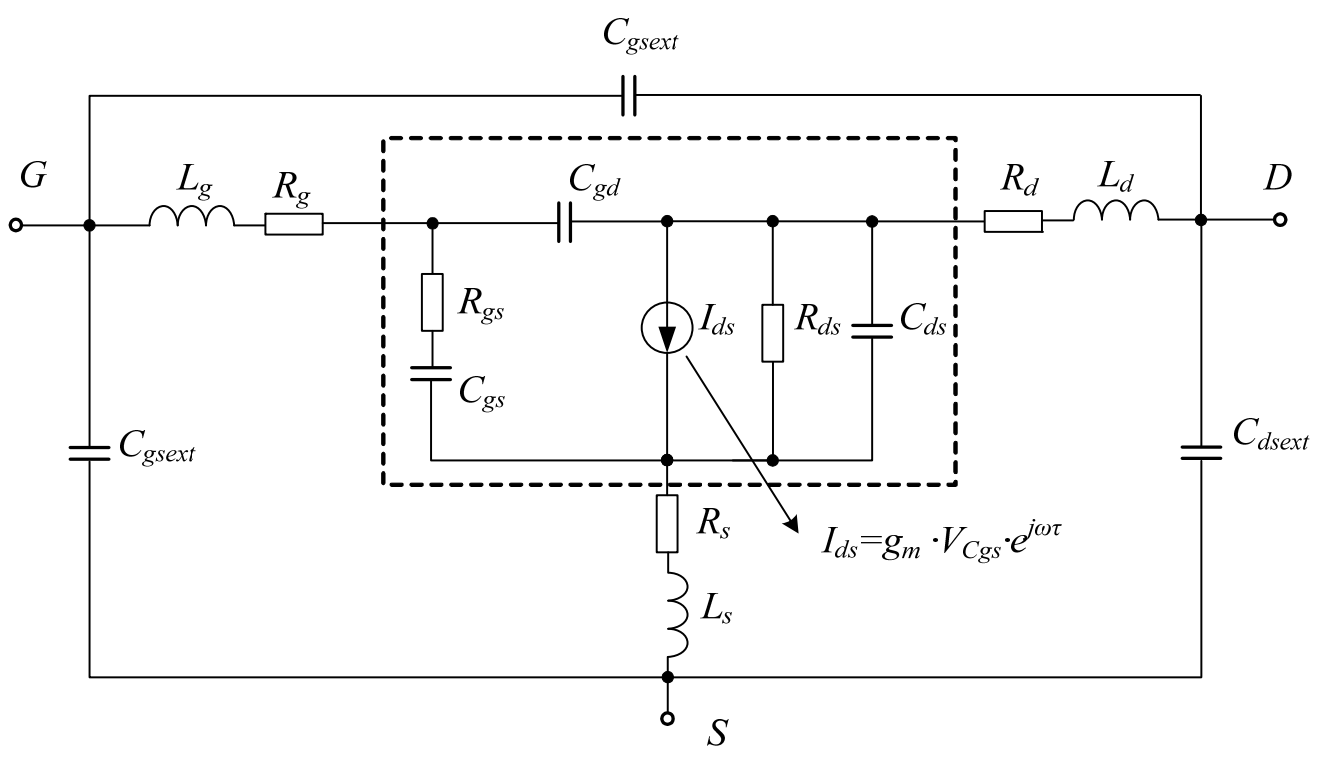

Рисунок 2 - Обобщенная малосигнальная модель полевого транзистора.

Модель состоит из внутренней и внешней частей, представляющих сам транзистор и элементы его корпуса. Внутренняя часть, выделенная на рис. 2 штриховой линией, для большинства предложенных малосигнальных моделей одинакова, однако, существуют модификации обобщенной модели. 
Во внутреннюю часть входят следующие элементы:

$R_{g s}\left(R_{i}\right)$ - сопротивление затвор-исток; зарядовое сопротивление, которое представляет резистивный путь для заряда емкости $C_{g s}$;

$R_{d s}$ - сопротивление сток-исток; выходное дифференциальное сопротивление;

$C_{g s}$ - емкость затвор-исток;

$C_{g d}$ - емкость затвор-сток; вместе с емкостью $C_{g s}$ моделируют заряд обедненной области под затвором;

$C_{d s}$ - емкость сток-исток; емкость подложки между стоком и землей;

$I_{d s}$ - источник тока стока, управляемый напряжением на емкости затвор-исток $C_{g s}$;

$g_{m}$ - крутизна;

$\tau$ - постоянная времени частотной зависимости источника тока.

Внешние паразитные элементы малосигнальной модели, изображенной на рис. 2, представлены индуктивностями $L_{g}, L_{s}, L_{d}$, сопротивлениями $R_{g}, R_{s}, R_{d}$ и емкостями $C_{g s e x t}$, $C_{\text {dsext }}, C_{\text {gdext }}$.

Определение (экстракцию) элементов линейной модели СВЧ транзистора обычно выполняют на основе измерения его малосигнальных параметров рассеяния при заданном смещении в требуемом диапазоне частот. Для экстракции параметров нелинейной модели, кроме малосигнальных параметров рассеяния, используются также измеренные вольтамперные и (или) вольтфарадные характеристики. Экстракция параметров нелинейных моделей значительно сложнее, однако хорошая компактная нелинейная модель описывает множество физических эффектов (влияние уровня сигнала, напряжений смещения, явления саморазогра, «trapping» эффекты и т.д.).

Физические компактные модели создаются в результате анализа физических процессов, протекающих в полупроводниковом приборе. При этом используются фундаментальные принципы работы устройства в качестве основы для феноменологических уравнений, описывающих поведение транзистора относительно его зажимов при помощи качественных и количественных физических макроскопических параметров. В качестве параметров могут выступать следующие величины: толщина активного полупроводникового слоя, длина затвора, легирование активного слоя, подвижность электронов, толщина оксидного слоя затвора и т.д. При этом допускаются некоторые допущения, например, распределение легирующих примесей может быть одномерным, квазидвумерным или квазитрехмерным. При получении таких моделей используется множество других упрощающих предположений о диапазоне применимости, погрешности аппроксимации, постоянстве параметров и др. Классическими примерами такого подхода являются модель полевого транзистора JFET [13] для кремниевых транзисторов, нелинейная модель НЕМТтранзистора для технологии ED02AH фирмы Ommic [14], серия моделей для MOSFET транзисторов BSIM [15] и др.

Недостатками указанных моделей являются сложность построения уравнений модели, большое количество извлекаемых параметров (у модели BSIM4 - больше 400 параметров), слабо формализованная процедура экстракции, привязанность к одной технологии изготовления; кроме того скорость моделирования ниже, чем для формальных моделей. Достоинствами являются высокая точность и «физичность» модели, т.е. связь с технологией, что позволяет оперативно изменять параметры модели при изменении параметров технологического процесса.

И формальные, и физические компактные модели транзисторов имеют схожие недостатки:

1) процесс подбора коэффициентов аппроксимационных функций является весьма трудоемким, нецеленаправленным и долгим;

2) часто оказывается, что указанные нелинейные модели в принципе не могут с достаточной точностью одновременно воспроизвести ВАX и малосигнальные параметры рассеяния конкретного транзистора при различных напряжениях смещения [16].

В табл. 1 приведены распространенные типы нелинейных моделей СВЧ ПТ, используемые в настоящее время разработчиками, и их основные характеристики.

Для описания MESFET и HEMT транзисторов наибольшее распространение в коммерческих САПР получили такие виды моделей как Angelov2, Curtice3, ТОМ3 и EЕНЕМТ. Как следует из табл. 1, фактически все они имеют одинаковый набор особенностей и учитываемых явлений (цепь для моделирования саморагозрева может быть легко добавлена).

В связи со сказанным сравнительно недавно были предложены универсальные (табличные) нелинейные модели СВЧ транзисторов в виде ЭС [17], которые строятся на основе непосредственно измеренных параметров прибора на постоянном токе и на высоких частотах (малосигнальные параметры рассеяния). В этих моделях используется аппроксимация нелинейных зависимостей элементов ЭС (источников тока и заряда) с помощью полиномов или сплайнов. Так как в конечном итоге используется модель в виде эквивалентной схемы и для получения нелинейных зависимостей не используются физических уравнения, протекающих в транзисторе процессов, логично 
рассматривать этот класс моделей как разновидность формальных компактных моделей.

Таблица 1

Основные нелинейные модели СВЧ ПТ и их свойства

\begin{tabular}{|c|c|c|c|c|c|c|}
\hline & JFET & Curtice3 & EEHEMT & Angelov2 & TOM3 & CMC \\
\hline $\begin{array}{c}\text { Вид СВЧ } \\
\text { транзистора }\end{array}$ & MESFET & MESFET & HEMT & $\begin{array}{c}\text { MESFET/ } \\
\text { HEMT }\end{array}$ & MESFET & MOSFET \\
\hline $\begin{array}{c}\text { Количество } \\
\text { параметров }\end{array}$ & 27 & 59 & 68 & 80 & 54 & 55 \\
\hline $\begin{array}{c}\text { Зарядовая } \\
\text { модель }\end{array}$ & - & + & + & + & + & + \\
\hline $\begin{array}{c}\text { Низкочастотная } \\
\text { дисперсия }\end{array}$ & - & + & + & + & + & + \\
\hline $\begin{array}{c}\text { Электрический } \\
\text { пробой }\end{array}$ & - & + & + & + & + & + \\
\hline Саморазгорев & - & - & - & + & - & + \\
\hline $\begin{array}{c}\text { Эффекты } \\
\text { ловушек }\end{array}$ & - & - & - & - & + & + \\
\hline $\begin{array}{c}\text { Температурная } \\
\text { модель }\end{array}$ & + & + & + & + & + & + \\
\hline
\end{tabular}

Идея табличных моделей получила широкое распространение и применялась в $[1,17,18]$. Достоинствами таких моделей являются высокая точность в широком диапазоне изменения частоты, уровня сигнала и режимов работы транзистора, простота экстракции, малое число параметров. Кроме того, процедура построения универсальной модели в принципе может быть формализована и автоматизирована. К недостаткам относятся: невозможность экстраполяции характеристик транзистора за пределами диапазона изменения параметров, для которого строилась модель; сложность обеспечения связи результатов моделирования с параметрами технологического процесса и геометрией прибора; необходимость уделять специальное внимание вопросам сходимости нелинейного анализа при использовании метода гармонического баланса.

Поведенческая модель (behavioral model) это модель в виде «черного ящика», в которой используются некоторые математические функции или преобразования для описания связи входных и выходных данных, последние обычно получаются экспериментально либо при использовании более детальных (физикотехнологических или компактных) моделей. Поведенческие модели являются формальными, т.е. строятся на основе формального сходства между поведением модели и объекта относительно внешних входов. При этом вид описывающих функций выбирается не на основе анализа физических процессов, происходящих в полупроводниковом приборе, а из условий быстродействия и требуемой точности воспроизведения взаимосвязи входных и выходных переменных. Таким образом, поведенческие модели никак не отражают внутреннюю структуру и процессы в приборе, в связи с этим в отечественной литературе используется также термин «бесструктурные модели» [5].

Наиболее простой поведенческой моделью, используемой при проектировании линейных активных СВЧ устройств, являются малосигнальные параметры транзистора как четырехполюсника - чаще всего S-параметры. Они могут быть получены путем непосредственных измерений либо расчетом по линейной ЭС. Для проектирования устройств, работающих в умеренно нелинейном режиме, используют большесигнальные параметры рассеяния (БСПР). При таком подходе транзистор (нелинейный прибор) фактически заменяется на заданной частоте в некоторой окрестности установившегося режима линейным эквивалентным четырехполюсником [19]. Также в качестве поведенческих моделей для проектирования УМ часто используются нагрузочные характеристики транзистора (так называемые load-pull измерения), модели в виде нейронных сетей и др.

Дальнейшим развитием идеи «черного ящика» являются X-параметры, разработанные Вершпехтом [20] и Рутом [21], сотрудниками компании Agilent (теперь Keysight). X-параметры - это математическое расширение S-параметров; они описывают нелинейную цепь в режиме малого и большого сигнала, интермодуляционные искажения, коэффициент передачи по мощности и т.п. Но определение Х-параметров требует проведения большого объема измерений при 
изменении в заданных диапазонах уровней и частот возбуждающих сигналов, постоянных напряжений на электродах транзистора и т.д. Сказанное определяет недостатки большесигнальных $\mathrm{S}$-параметров и $\mathrm{X}$ параметров по сравнению с нелинейными эквивалентными схемами. Более подробно большесигнальные S- и X-параметры описаны в [21].

Последними и самыми оригинальными являются аппаратные модели - модели в виде устройства, которое заменяет исследуемый транзистор при моделировании и расчете СВЧ схем. По сути это метод макетирования, т.е. вместо математических моделей при проектировании используется реальный транзистор. Однако в отличие от макетирования, в аппаратных моделях используются современные достижения в компьютерной технике - моделирование отдельных частей модели в САПР, регулировка параметров модели, встраивание в стандартные программы схемотехнического моделирования [2]. Обычно аппаратная модель делится на две части статическая и динамическая. Статическая часть (BAX) моделируется при помощи реального транзистора, а динамическая (нелинейные емкости и т.п.) при помощи стандартных формальных компактных моделей [2].

Достоинством моделей такого типа является то, что устраняется этап экстракции параметров и этап разработки уравнений модели, которые являются наиболее мощными источниками ошибок. Недостатками являются громоздкость, сложность в эксплуатации, отсутствие «готового решения» и непопулярность среди инженеров.

Заключение. В работе выполнен обзор используемых в настоящее время типов моделей полевых и НЕМТ транзисторов, основанный на общей классификации моделей полупроводниковых приборов. Обзор может быть полезен для специалистов по проектированию СВЧ-устройств при выборе моделей СВЧтранзисторов, а также в качестве основы для разработки своей собственной методики экстракции параметров нелинейной модели.

\section{References:}

1. Aaen P, Pla J, Wood J (2007) Modeling and Characterization of RF and Microwave power FETs. Cambridge University Press, 267.

2. Denisenko V (2004) Modelirovanie MOP tranzistorov - metodologicheskiy aspekt. zhurnal «Komponenty i tekhnologii», No.7, pp.56-61. \& No.9, pp.32-39.

3. Reiser M (1972) Large-scale numerical simulation in semiconductor device modeling. Comput. Methods in App. Mech. Eng., No.1, pp.17-38.

4. Burtula EM, etc. (1981) Finite-element analysis of semiconductor devices: the FIELDAY program. IBM J. Res. Dev., 07/1981, No.4, pp.218-231.

5. Shvarts NZ (1980) Lineynye tranzistornye usiliteli SVCh. Moscow: Sov. radio, 368.

6. Angelov I, Zirath H, Rorsman N (2002) New empirical nonlinear model for HEMT and MESFET devices. IEEE Trans. Microwave Theory Tech., 05/2002, Vol. 40, Dec. 1992, pp.2258-2266.

7. Materka A, Kacprzak T (1985) Computer calculations of large-signal GaAs FET amplifier characteristic. IEEE Trans. Microwave Theory Tech., 02/1985, vol. 33, pp.129-135.

8. Curtice W, Ettenberg M (1985) A nonlinear GaAs FET model for use in the design of output circuit for power amplifier. IEEE Trans. MTT. Vol. MTT-33. No 12. pp. 1383-1394.
9. (2014) Agilent Technoligies. Available: http://www.home.agilent.com/ru/pc-

1297149/ic-cap-device-modelingsoftwaremeasurement-control-and-parameterextraction (accessible 15.11.2014).

10. Kokolov AA, Babak LI (2010) Metodika postroeniya nelineynoy modeli EEHEMT dlya geterostrukturnykh polevykh SVChtranzistorov. Doklady TUSUR. Izdatel'stvo TUSURa. Ch. 2 (22), pp.149-152.

11. (2014) AmCAD Engineering. Available: http://www.amcadengineering.ru/Modelirovanie-

PolevyhTranzistorov.html

(accessible 15.11.2014).

12. Dambrine G, etc. (1988) A new method for determining the FET small-signal equivalent circuit. IEEE Trans. Microwave Theory Tech., 07/1988, Vol.36, No.7, pp.1151-1159.

13. Shockley W (1952) A unipolar field-effect transistor. Proc. IRE, vol.40, pp.1365-1376.

14. Ommic. Available: http://www.ommic.com/site/tech-2 (accessible 15.11.2014).

15. Cheng $\mathrm{Y}, \mathrm{Hu} \mathrm{C}$ (1999) MOSFET modeling \&BSIM3 user's guide. Kluwer Academic Publishers, 461.

16. Klimova AV, Korolev AN, etc. (2006) Sravnenie nelineynykh modeley dlya 
tranzistorov s submikronnym zatvorom. Radiotekhnika, 2006, No.3, pp. 72-77.

17. Root DE, Fan S, Meyer J (1991) Technology independent large-signal non quasistatic FET models by direct construction from automatically characterized device data. Proc. 21st Eur. Microwave Conf., 1991, pp.927-932.

18. Curras-Francos MC (2005) Table-based nonlinear HEMT model extracted from timedomain large-signal measurements. Microwave Theory and Techniques, IEEE Transactions on, Vol. 53, Issue 5, May 2005, pp. 1593-1600.
19. Colantonio P, Giannini F, Leuzzi G, Limiti E (1995) Direct-synthesis design technique for nonlinear microwave circuits. IEEE Trans. MTT. Vol. MTT-43. No. 12, pp. 2851-2855.

20. Verspecht J, Williams DF, Shreurs D, Remley KA, McKinley MD (2005) Linearization of large signal scattering functions. IEEE Trans. MTT. Vol. MTT-53. -No. 4, pp. 1369-1376.

21. Verspecht J., Root D.E. (2006) Polyharmonic distortion modeling. IEEE Microwave Magazine. Vol. 7, No. 3. pp.44-57. 